\title{
Nutritional risk in allogeneic stem cell transplantation: Rationale for a tailored nutritional pathway
}

Takashi Aoyama ${ }^{1}$, Osamu Imataki ${ }^{2}$, Keita Mori ${ }^{3}$, Kanako Yoshitsugu ${ }^{4}$, Masafumi Fukaya ${ }^{4}$, Terukazu Enami ${ }^{4}$, Ikue Okamura ${ }^{4}$, Raine Tatara ${ }^{4}$, Takashi Ikeda ${ }^{4}$ 1 Division of Nutrition, Shizuoka Cancer Center, 2 Division of Hematology \& Stem Cell Transplantation,Kagawa University Hospital,

3 Division of Biostatistician, Shizuoka Cancer Center, 4 Division of Hematology \& Stem Cell Transplantation,Shizuoka Cancer Center

\section{Rationale}

Hematopoietic stem cell transplantation carries nutritional related risks; therefore, nutritional therapies needs to be immediately initiated after starting treatment. We hypothesized that the nutritional risk of allogeneic Blood stem cell Transplantation could be affected by nutrition adverse events, and we evaluated the usefulness of nutritional pathways involving nutritional intervention in comparison with those involving other post hoc nutritional intervention.

\section{Objectives}

We assess nutritional risk among patients who undergo allogeneic stem cell transplantation.

\section{Methods}

【Study design】A retrospective study. Post-hoc power analysis. 【Study period】 April. 2007 to Dec. 2012 【Sample size】 51 patients (Shizuoka Cancer Center, Japan) 【Evaluation period】The pretreatment of pretreatment for transplantation to after the end of Parenteral Nutrition (PN).

\section{Nutritional assessment point : $\mathrm{T} 1$ point to $\mathrm{T} 2$ point.}

$\star$ Nutritional assessment item.

$\bigcirc \mathrm{BMI}: \mathrm{T} 1 \quad \bigcirc$ Loss of Body Weight (LBW and \%) $: \mathrm{T} 1 \rightarrow \mathrm{T} 2 \stackrel{\ln B \text { ody } 520}{ }$

$\bigcirc$ BIA ; In Body S20 ®(Biospace) : T1 $\rightarrow$ T2

$2 \mathrm{~h}$ after the patient's breakfast.

Extracellular fluid ; ECF/ total body fluid ;TBF : $0.38 \downarrow$

Extracellular water ;ECW/ total body water TBW : $0.40 \downarrow$

$\bigcirc$ Relationship between: $\mathrm{T} 1 \rightarrow \mathrm{T} 2$

Nutrient supply (calorie, protein / IBW ) and weight ,BIA (SMM, FM).

"\%LBW $\geq 7.5$ " group vs "\%LBW $<7.5$ " group (Cut off \%7.5LBW : 3 month ; Jane V.JPEN, 2012.)

BMI, IBW\%, LBW\%, BIA, Nutrient supply (calorie ,protein / IBW ), Start day of oral intake, and engraftment day graft versus host disease; GVHD

$\bigcirc$ Relationship between: $\mathrm{T} 1 \rightarrow \mathrm{T} 2 \mathrm{CTCAE}$ and orally ingested calories

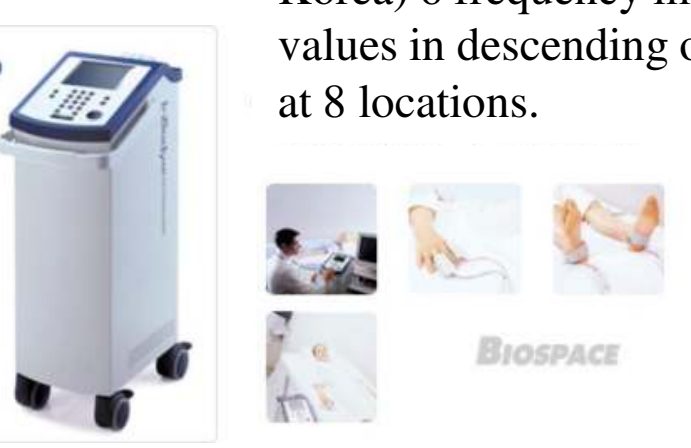
at 8 locations.

Table 1. Nutritional assessment

\begin{tabular}{lll}
\hline & & P value* \\
\hline Preoperative BMI (min-max) & $22.1 \mathrm{~kg} / \mathrm{m}^{2}(16.5-31.5)$ & \\
\hline Assessment period (min-max) & $54 \mathrm{days}(29-98)$ & \\
\hline$\% \mathrm{LBW}$ (min-max) & $-4.5 \%(4.9--18.8)$ & \\
\hline Significant \%LBW cases (n) & $13 / 51$ & \\
\hline Preoperative body weight (min-max) & $62.6 \mathrm{~kg}(40.3-92.3)$ & \\
\hline Weight variations during assessment period (min-max) & $-2.5 \mathrm{~kg}(2.5--14.1)$ & $<0.0001$ \\
\hline Preoperative skeletal muscle mass (min-max) & $25.3 \mathrm{~kg}(13.5-34.5)$ & \\
\hline Skeletal muscle mass variations during the assessment period (min-max) & $-1.2 \mathrm{~kg}(1.8--7.4)$ & $<0.0001$ \\
\hline Preoperative body fat mass (min-max) & $16.2 \mathrm{~kg}(3.7-33.9)$ & \\
\hline Body fat mass variations during the assessment period (min-max) & $-0.7 \mathrm{~kg}(-1.5--3.0)$ & 0.0004 \\
\hline Phase angle variations during the assessment period & $-0.66^{\circ}(0.7--3.65)$ & $<0.0001$ \\
\hline Pretreatment BIA (min-max): ECF/TBF, & $0.35(0.31-0.37)$ & \\
ECW/TBW (min-max) & $0.39(0.36-0.42)$ & \\
\hline Post-PN off BIA (min-max): ECF/TBF, & $0.35(0.33-0.37)$, & \\
ECW/TBW (min-max) & $0.40(0.37-0.42)$ & \\
\hline Total calorie supply (min-max) & $23 \mathrm{kcal} / \mathrm{IBW} / \mathrm{day}(17-34)$ & \\
\hline PN calorie supply sufficiency rate (min-max) & $53 \%(0-82)$ & \\
\hline Orally ingested calorie supply sufficiency rate (min-max) & $47 \%(18-100)$ & \\
\hline EN calorie supply sufficiency rate (min-max) & $0 \%(0)$ & \\
\hline
\end{tabular}

Table 2. Number of participants by PS assessment results

\begin{tabular}{lccccc}
\hline & Grade 0 & Grade 1 & Grade 2 & Grade 3 & Grade 4 \\
\hline Preoperative PS & 51 & 0 & 0 & 0 & 0 \\
\hline Max PS grade during assessment period & 0 & 0 & 18 & 31 & 2 \\
\hline
\end{tabular}

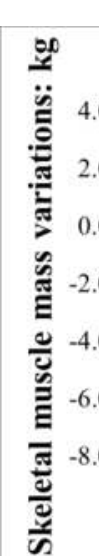

Weight and skeletal muscle mass variations of all subjects $(r=0.89, P<0.0001)$

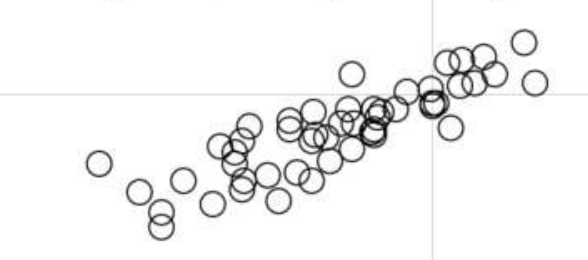

$$
-10.0 \quad-5.0 \quad 0.0
$$

Weight variations and calorie supply of all subjects $(\mathrm{r}=0.517, \mathrm{P}=0.0001)$

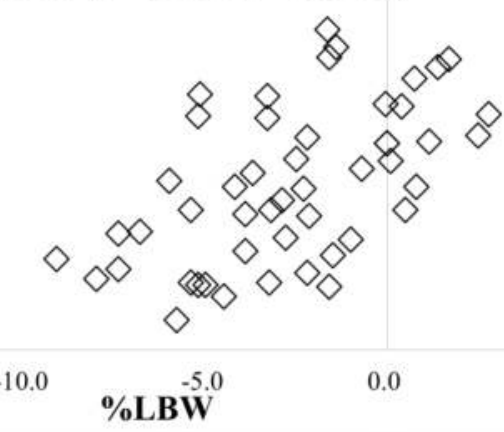

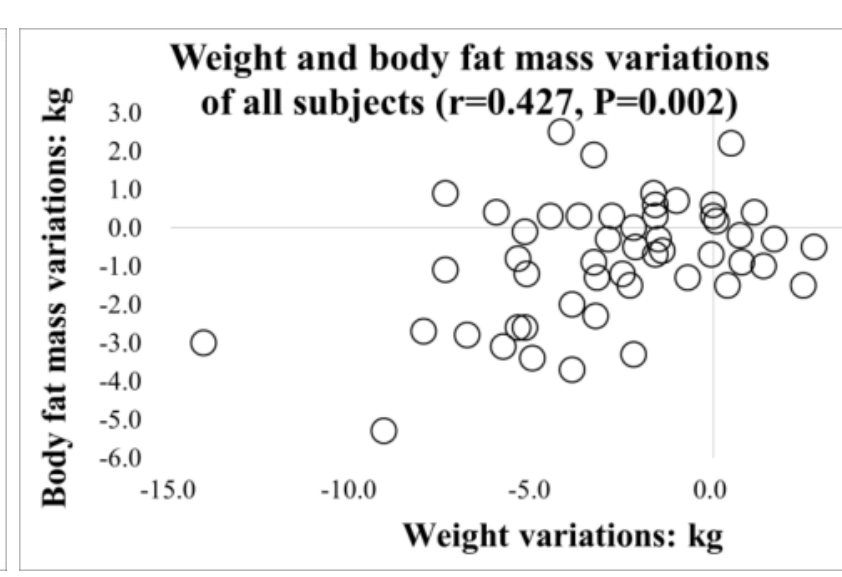

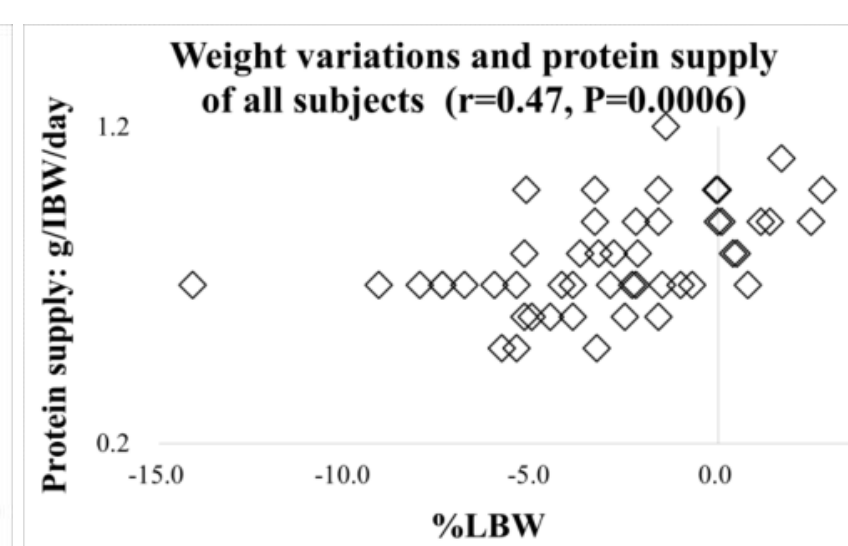
In Body S20 body (Biospace, Korea) 6 frequency impedance values in descending order

Results

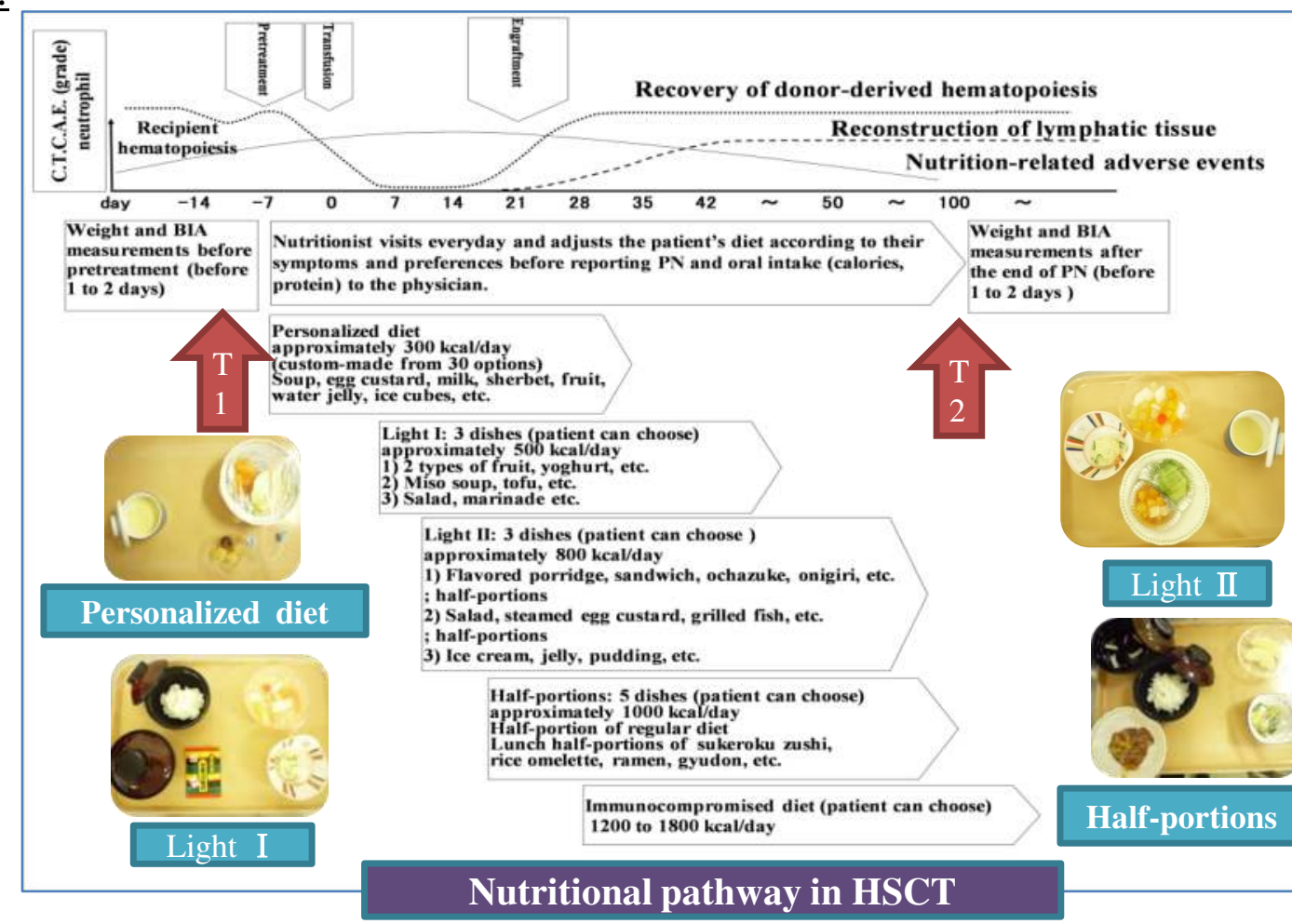

Table 3. Comparisons between the $\% \mathrm{LBW} \geq 7.5 \%$ group and the $\% \mathrm{LBW}<7.5 \%$ group

\begin{tabular}{|c|c|c|c|}
\hline & $\begin{array}{c}\% \text { \%BW } \geq 7.5 \text { Group } \\
(\mathrm{n}=13)\end{array}$ & $\begin{array}{c}\text { \%LBW }<7.5 \text { Group } \\
(\mathrm{n}=38)\end{array}$ & P value* \\
\hline Age (min-max) & $51(17-66)$ & $52(17-68)$ & 0.34 \\
\hline Preoperative BMI (min-max) & $23.3(18.7-28.3)$ & $21.9(16.9-31.5)$ & 0.27 \\
\hline \multicolumn{4}{|l|}{ Disease } \\
\hline AML & 5 & 9 & \multirow{6}{*}{0.524} \\
\hline MDS & 3 & 9 & \\
\hline CML & 1 & 4 & \\
\hline ALL & 2 & 5 & \\
\hline ML & 0 & 8 & \\
\hline Other & 2 & 3 & \\
\hline \multicolumn{4}{|l|}{ Pretreatment regimen } \\
\hline $\mathrm{Bu} / \mathrm{CY}$ & 3 & 9 & \multirow{4}{*}{0.976} \\
\hline $\mathrm{CY} / \mathrm{TBI}$ & 2 & 5 & \\
\hline $\mathrm{Fu} / \mathrm{Mel}$ & 5 & 13 & \\
\hline $\mathrm{Fu} / \mathrm{Bu}$ & 3 & 11 & \\
\hline \multicolumn{4}{|l|}{ Donor relation } \\
\hline Related & 10 & 26 & \multirow[t]{2}{*}{0.561} \\
\hline Unrelated & 3 & 12 & \\
\hline Assessment period (min-max) & 61 days (29-98) & 55 days (29-91) & 0.37 \\
\hline$\%$ IBW (min-max) & $106 \%(90-129)$ & $100 \%(77-143)$ & 0.28 \\
\hline \%LBW (min-max) & $-9.0 \%(-8.1-18.8)$ & $-3.1 \%(-4.9-6.8)$ & $<0.0001$ \\
\hline Total calorie supply (min-max) & $20 \mathrm{kcal} / / \mathrm{BBW} /$ day $(18-28)$ & $24 \mathrm{kcal} / / \mathrm{BB} / \mathrm{day}(17-34)$ & 0.01 \\
\hline PN calorie supply (min-max) & $13 \mathrm{kcal} / \mathrm{IBW} / \mathrm{day}(0-20)$ & $11 \mathrm{kcal} / \mathrm{IBW} / \mathrm{day}(0-21)$ & 0.59 \\
\hline Orally ingested calorie supply (min-max) & $8 \mathrm{kcal} / \mathrm{IBW} / \mathrm{day}(4-22)$ & $12 \mathrm{kcal} / \mathrm{IBW} / \mathrm{day}(4-28)$ & 0.01 \\
\hline Total protein supply (min-max) & $0.7 \mathrm{~g} / \mathrm{IBW} / \mathrm{day}(0.5-1.0)$ & $0.8 \mathrm{~g} / \mathrm{IBW} / \mathrm{day}(0.5-1.4)$ & 0.02 \\
\hline PN protein supply (min-max) & $0.4 \mathrm{~g} / \mathrm{IBW} / \mathrm{day}(0-0.7)$ & $0.4 \mathrm{~g} / \mathrm{IBW} / \mathrm{day}(0-0.7)$ & 0.73 \\
\hline Orally ingested protein supply (min-max) & $0.3 \mathrm{~g} / \mathrm{IBW} / \mathrm{day}(0.1-0.7)$ & $0.4 \mathrm{~g} / \mathrm{IBW} / \mathrm{day}(0.2-1.1)$ & 0.009 \\
\hline Start day of oral intake (min-max) & Day $21(0-53)$ & Day $13(0-53)$ & 0.04 \\
\hline Engraftment day (min-max) & Day 18 (12-27) & Day 16 (9-27) & 0.17 \\
\hline Acute GVHD incidence & $79.9 \%(10 / 13)$ & $42.1 \%(16 / 38)$ & 0.03 \\
\hline Acute GVHD-skin max stage (min-max) & $1.3(0-3)$ & $0.7(0-3)$ & 0.0428 \\
\hline Acute GVHD-liver max stage (min-max) & $0.0(0-1)$ & $0.0(0)$ & 0.5893 \\
\hline Acute GVHD-intestine max stage (min-max) & $0.7(0-3)$ & $0.1(0-1)$ & 0.0138 \\
\hline Acute GVHD-grade (min-max) & $1.3(0-3)$ & $0.5(0-1)$ & 0.0095 \\
\hline
\end{tabular}

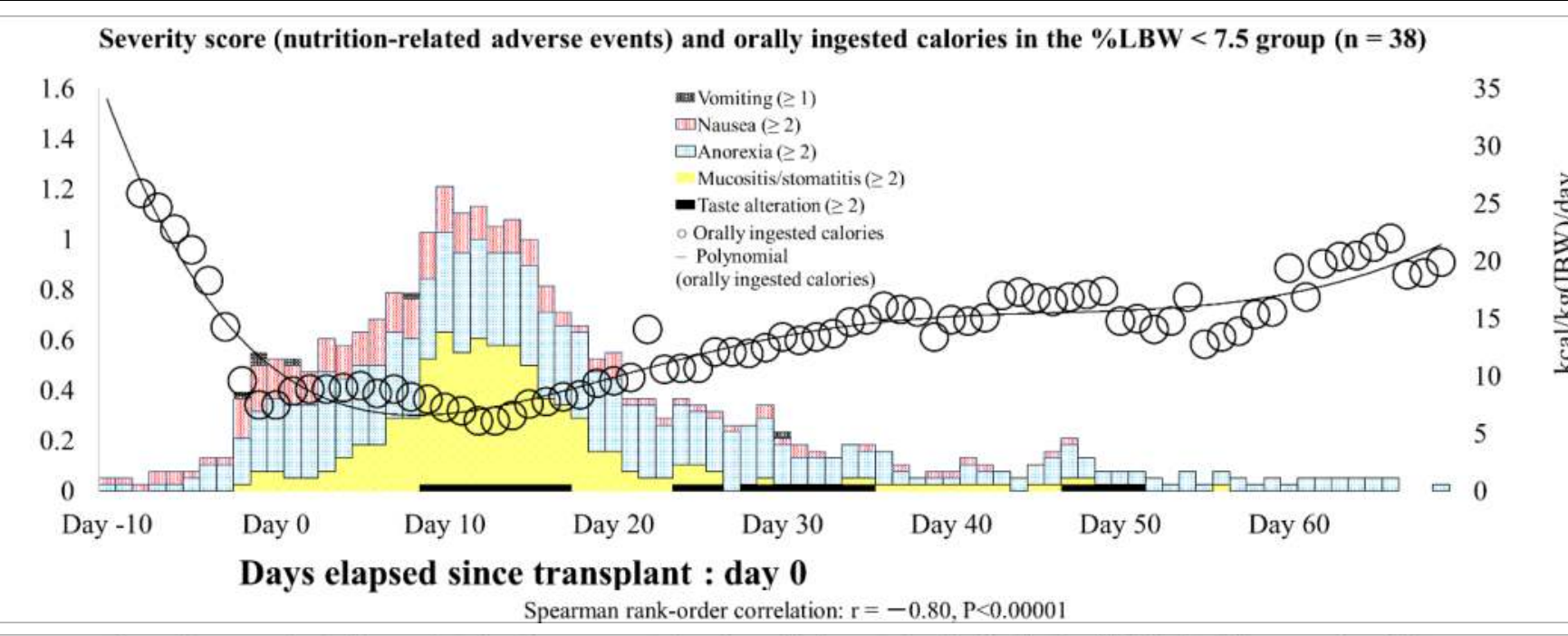

Spearman rank-order correlation: $r=-0.80, P<0.00001$

Severity score (nutrition-related adverse events) and orally ingested calories in the $\% \mathrm{LBW} \geq 7.5$ group $(\mathrm{n}=13)$

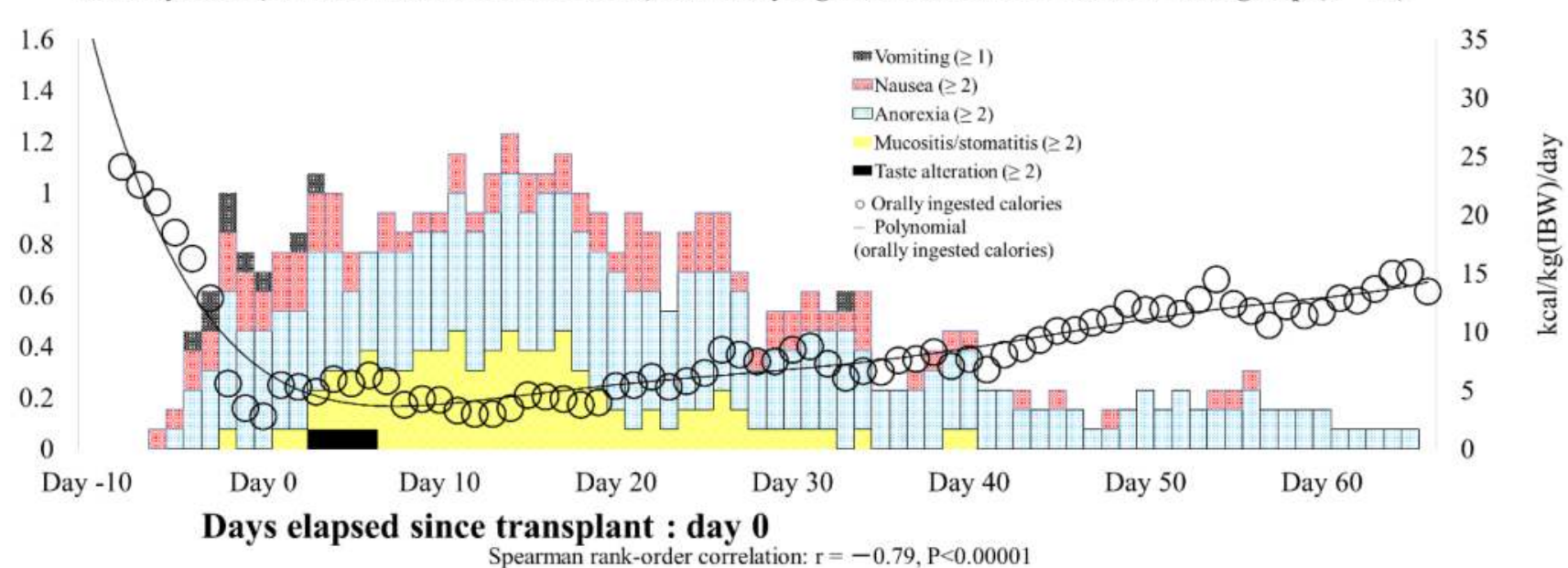

Conclusion

I think Early and customized nutritional intervention may be optimal for all patients who undergo allogeneic stem cell transplantation to ameliorate body weight loss associated with nutritionrelated adverse events, intestinal GVHD and nutritional status. 